\title{
Uniqueness of Nash equilibria in a quantum Cournot duopoly game
}

\author{
Yohei Sekiguchi $^{1}$, Kiri Sakahara ${ }^{1}$ and Takashi Sato ${ }^{1,2}$ \\ ${ }^{1}$ Graduate School of Economics, University of Tokyo, 7-3-1 Hongo, Bunkyo-ku, \\ Tokyo 113-0033, Japan \\ ${ }^{2}$ Faculty of Economics, Toyo University, 5-28-20 Hakusan, Bunkyo-ku, Tokyo 112-8606, \\ Japan \\ E-mail: yohei@e.u-tokyo.ac.jp, equirit@mail.ecc.u-tokyo.ac.jp and tksato@toyonet.toyo.ac.jp
}

Received 22 September 2009, in final form 22 February 2010

Published 18 March 2010

Online at stacks.iop.org/JPhysA/43/145303

\begin{abstract}
A quantum Cournot game whose classical form game has multiple Nash equilibria is examined. Although the classical equilibria fail to be Pareto optimal, the quantum equilibrium exhibits the following two properties: (i) if the measurement of entanglement between strategic variables chosen by the competing firms is sufficiently large, the multiplicity of equilibria vanishes, and (ii) the more strongly the strategic variables are entangled, the more closely the unique equilibrium approaches to the optimal one.
\end{abstract}

PACS numbers: 03.67.-a, 02.50.Le

(Some figures in this article are in colour only in the electronic version)

\section{Introduction}

Game theory is a powerful mathematical tool to analyze various natural and social phenomena [1-3]. After the publication of Meyer [4], there has been a great deal of effort to extend the classical game theory into the quantum domain, and it has been shown that quantum games may have significant advantages over their classical counterparts [4-6]. The classical game theory has a fatal drawback, namely the multiplicity of equilibria. Battle of sexes, chicken game and stag hunt are famous examples of games with multiple equilibria. For a game possessing multiple equilibria, the classical game theory can say nothing about the predictability of the outcome of the game: there is no particular reason to single one out of these equilibria. Until now, several quantum extensions are considered to resolve this problem, e.g. battle of sexes [7-13], chicken game [14-17] and stag hunt [16-18]. Du et al [19, 20] analyzed the relation between the entanglement parameter and the number of equilibria in quantum prisoners' dilemma, and found thresholds in the entanglement parameter where the 
number of equilibria changes. We also attack this problem by analyzing a quantum extension of a game which describes market competition.

In economics, many important markets are neither perfectly competitive nor perfectly monopolistic, that is, the action of individual firms affects the market price [21]. These markets are usually called oligopolistic and can be analyzed based on game theory. Recently, Li et al [22] investigated the quantization of games with continuous strategic space, a classic instance of which is the Cournot duopoly [23], in which firms compete on the amount of output they will produce, which they decide on independently of each other and at the same time. Li et al [22] showed that the firms can escape the frustrating dilemma-like situation if the structure involves a maximally entangled state. A key feature in [22] is the linearity assumption, that is, both the cost function and the inverse demand function are linear. It is well known that linear Cournot games have exactly one equilibrium [21]. On the other hand, in nonlinear settings, there may be multiple equilibria, and hence we may not predict the market price. A natural question is whether the uniqueness of equilibria is guaranteed in the quantum Cournot duopoly. We are trying to answer this question in this paper. To quantize the model, we apply Li et al's [22] 'minimal' quantization rules to Cournot duopoly in a nonlinear setting, where there are one symmetric equilibrium and two asymmetric equilibria in the zero entanglement case ${ }^{3}$. We observe the transition of the game from purely classical to fully quantum, as the game's entanglement increases from zero to maximum. We show that if the entanglement of the game is sufficiently large, then all asymmetric equilibria vanish and there remains one symmetric equilibrium. Furthermore, similar to Li et al [22], in the maximally entangled game, the unique symmetric equilibrium is exactly Pareto optimal. In other words, the multiplicity of equilibria as well as the dilemma-like situation in the classical Cournot duopoly is completely resolved in our quantum extension.

\section{Classical Cournot duopoly}

We consider Cournot competition between two firms, firm 1 and firm 2 . They simultaneously decide the quantities $q_{1}$ and $q_{2}$, respectively, of a homogenous product they want to put on the market. Let $P(Q)$ be the inverse demand function, where $Q=q_{1}+q_{2}$. Each firm $j \in\{1,2\}$ has the common cost function $C\left(q_{j}\right)$. Then the firm $j$ 's profit can be written as

$$
u_{j}\left(q_{1}, q_{2}\right)=P(Q) q_{j}-C\left(q_{j}\right) .
$$

We assume that

$$
\begin{aligned}
& P(Q)=a+b-Q, \\
& C\left(q_{j}\right)=\frac{1}{4}\left(q_{j}-a\right)^{4}-q_{j}^{2}+b q_{j}-d,
\end{aligned}
$$

where $a, b, d>0$.

Given any $q_{2}$, we have $\partial^{2} u_{1}\left(q_{1}, q_{2}\right) / \partial q_{1}^{2} \leqslant 0$. Thus, to maximize her profit, firm 1 chooses $q_{1}$ such that $\partial u_{1} / \partial q_{1}=0$, that is,

$$
-q_{2}-\left(q_{1}-a\right)^{3}+a=0
$$

Similarly, given any $q_{1}$, firm 2 chooses $q_{2}$ such that

$$
-q_{1}-\left(q_{2}-a\right)^{3}+a=0 .
$$

3 Several quantum extensions of oligopolistic competition, applying Li et al's [22] 'minimal' quantization rules, have been considered, e.g. the quantum Cournot duopoly game [24, 25], the quantum Bertrand duopoly game $[26,27]$, the quantum Stackelberg duopoly game $[28,29]$ and the quantum oligopoly game [30]. 
A pair $\left(q_{1}, q_{2}\right)$ is a Nash equilibrium iff it solves equations (4) and (5). Then, there are three equilibria, $\left(q_{1}, q_{2}\right)=(a, a),(a-1, a+1)$ and $(a+1, a-1)$. At these equilibria the profits are

$\left(u_{1}\left(q_{1}, q_{2}\right), u_{2}\left(q_{1}, q_{2}\right)\right)= \begin{cases}(d, d) & \text { if }\left(q_{1}, q_{2}\right)=(a, a) \\ (3 / 4-a+d, 3 / 4+a+d) & \text { if }\left(q_{1}, q_{2}\right)=(a-1, a+1) \\ (3 / 4+a+d, 3 / 4-a+d) & \text { if }\left(q_{1}, q_{2}\right)=(a+1, a-1)\end{cases}$

However, these equilibria fail to be Pareto optimal. The reason why they fail is that both firms can be better off by jointly decreasing their outputs, since $\partial u_{i} / \partial q_{i}=0$ and $\partial u_{i} / \partial q_{j}=-q_{i}<0$ at equilibria. On the other hand, if the two firms can cooperate and restrict their quantities, then they can increase their joint profit $u_{1}+u_{2}$. In particular, the joint profit is maximized at $\left(q_{1}^{*}, q_{2}^{*}\right)$ such that $\partial\left(u_{1}\left(q_{1}^{*}, q_{2}^{*}\right)+u_{2}\left(q_{1}^{*}, q_{2}^{*}\right)\right) / \partial q_{i}=0$ for $i=1,2$, that is,

$$
q^{*} \equiv q_{1}^{*}=q_{2}^{*}=a+2 \alpha \beta^{-1}-\frac{1}{2} \alpha^{2} \beta,
$$

where $\alpha \equiv(2 / 3)^{1 / 3}$ and $\beta \equiv\left(9 a+\sqrt{96+81 a^{2}}\right)^{1 / 3}$. (Obviously $\left(q^{*}, q^{*}\right)$ is Pareto optimal.) For example,

$$
u_{1}\left(q^{*}, q^{*}\right)=u_{2}\left(q^{*}, q^{*}\right)=7 / 4+d
$$

for $a=3$. Thus, the joint profit at $\left(q^{*}, q^{*}\right)$ is greater than that of any equilibrium.

With regard to the asymmetric equilibria, the situation is similar to that of a chicken game if we correspond the equilibria $(a-1, a+1),(a+1, a-1)$, respectively, to the equilibria (cooperate, defect), (defect, cooperate) in the chicken game. Below we will see that, as the measure of entanglement goes to infinitely large in a quantum form of Cournot competition, the unique equilibrium comes to be optimal, as if the unique cooperative equilibrium is attained in the chicken game [15].

\section{Quantum Cournot duopoly}

To model Cournot duopoly on a quantum domain, we follow Li et al's 'minimal' extension, which utilizes two single-mode electromagnetic fields, the quadrature amplitudes of which have a continuous set of eigenstates. The tensor product of two single-mode vacuum states $|v a c\rangle_{1} \otimes|v a c\rangle_{2}$ is identified as the starting state of the Cournot game, and the state consequently undergoes a unitary entanglement operation $\hat{J}(\gamma) \equiv \exp \left\{-\gamma\left(\hat{a}_{1}^{\dagger} \hat{a}_{2}^{\dagger}-\hat{a}_{1} \hat{a}_{2}\right)\right\}$, in which $\hat{a}_{1}$ and $\hat{a}_{2}\left(\hat{a}_{1}^{\dagger}\right.$ and $\left.\hat{a}_{2}^{\dagger}\right)$ are the annihilation (creation) operators of the electromagnetic field modes. The operation is assumed to be known to both firms and to be symmetric with respect to the interchange of the two field modes. The resultant state is given by $\left|\psi_{i}\right\rangle \equiv \hat{J}(\gamma)|\mathrm{vac}\rangle_{1} \otimes|\mathrm{vac}\rangle_{2}$. Then firm 1 and firm 2 execute their strategic moves via the unitary operations $\hat{D}_{1}\left(x_{1}\right) \equiv$ $\exp \left\{x_{1}\left(\hat{a}_{1}^{\dagger}-\hat{a}_{1}\right) / \sqrt{2}\right\}$ and $\hat{D}_{2}\left(x_{2}\right) \equiv \exp \left\{x_{2}\left(\hat{a}_{2}^{\dagger}-\hat{a}_{2}\right) / \sqrt{2}\right\}$, respectively, which correspond to the quantum version of the strategies of the Cournot game. The final measurement is made, after these moves are finished and a disentanglement operation $\hat{J}(\gamma)^{\dagger}$ is carried out. The final state prior to the measurement, thus, is $\left|\psi_{f}\right\rangle \equiv \hat{J}(\gamma)^{\dagger} \hat{D}_{1}\left(x_{1}\right) \hat{D}_{2}\left(x_{2}\right) \hat{J}(\gamma)|\mathrm{vac}\rangle_{1} \otimes|\mathrm{vac}\rangle_{2}$. The measured observables are $\hat{X}_{1} \equiv\left(\hat{a}_{1}^{\dagger}+\hat{a}_{1}\right) / \sqrt{2}$ and $\hat{X}_{2} \equiv\left(\hat{a}_{2}^{\dagger}+\hat{a}_{2}\right) / \sqrt{2}$, and the measurement is done by the homodyne measurement with an infinitely squeezed reference light beam (i.e. the noise is reduced to zero). When quantum entanglement is not present, namely $\gamma=0$, this quantum structure faithfully represent the classical game, and the final measurement provides the original classical results: $q_{1} \equiv\left\langle\psi_{f}\left|\hat{X}_{1}\right| \psi_{f}\right\rangle=x_{1}$ and $q_{2} \equiv\left\langle\psi_{f}\left|\hat{X}_{2}\right| \psi_{f}\right\rangle=x_{2}$. Otherwise, namely when quantum entanglement is present, the quantities the two firms will produce are determined by 


$$
\begin{aligned}
& q_{1}=x_{1} \cosh \gamma+x_{2} \sinh \gamma, \\
& q_{2}=x_{2} \cosh \gamma+x_{1} \sinh \gamma .
\end{aligned}
$$

Note that the classical model can be recovered by choosing $\gamma$ to be zero, since the two firms can directly decide their quantities. On the other hand, both $q_{1}$ and $q_{2}$ are determined by $x_{1}$ and $x_{2}$ when $\gamma \neq 0$. It leads to the correlation between the firms.

Substituting $q_{j}$ into equation (1) provides the quantum profits $u_{j}^{Q}$ for firm $j$ :

$$
u_{j}^{Q}\left(x_{1}, x_{2}\right)=P\left(x_{1}, x_{2}\right)\left(x_{j} \cosh \gamma+x_{i} \sinh \gamma\right)-C\left(x_{j}, x_{i}\right),
$$

where $i \neq j$ and

$$
\begin{aligned}
P\left(x_{1}, x_{2}\right)= & a+b-e^{\gamma}\left(x_{1}+x_{2}\right), \\
C\left(x_{j}, x_{i}\right)= & \frac{1}{4}\left(x_{j} \cosh \gamma+x_{i} \sinh \gamma-a\right)^{4} \\
& \quad-\left(x_{j} \cosh \gamma+x_{i} \sinh \gamma\right)^{2}+b\left(x_{j} \cosh \gamma+x_{i} \sinh \gamma\right)-d .
\end{aligned}
$$

Similar to the classical game, we also have $\partial^{2} u_{j}\left(x_{j}, x_{i}\right) / \partial x_{j}^{2} \leqslant 0$ for any $x_{i}$. To maximize her profit, thus, firm $j$ chooses $x_{j}$ such that $\partial u_{j} / \partial x_{j}=0$, that is,

$$
-x_{j} \sinh 2 \gamma-x_{i} \cosh 2 \gamma-\left(x_{j} \cosh \gamma+x_{i} \sinh \gamma-a\right)^{3} \cosh \gamma+a \cosh \gamma=0 .
$$

Solving equation (14) for both firms provides the quantum Nash equilibria and the symmetric one is uniquely given as

$$
x^{*}(\gamma) \equiv x_{1}^{*}=x_{2}^{*}=\frac{a}{e^{\gamma}}+\operatorname{sech} \gamma \cdot \alpha \eta^{-1}-\frac{1}{2} \alpha^{2} \frac{\eta}{e^{\gamma}},
$$

where $\eta \equiv\left(9 a \tanh \gamma+\sqrt{12 e^{3 \gamma} \operatorname{sech}^{3} \gamma+81 a^{2} \tanh ^{2} \gamma}\right)^{1 / 3}$. As easily seen from equation (15), the quantity produced by each firm in the equilibrium, equal to $e^{\gamma} x^{*}(\gamma)$, monotonically increases and approaches to the Pareto optimal one $q^{*}$, as the entanglement $\gamma$ increases. In fact, given $\lim _{\gamma \rightarrow \infty} \tanh \gamma=1$ and $\lim _{\gamma \rightarrow \infty} e^{\gamma} \operatorname{sech} \gamma=2$, we have $\lim _{\gamma \rightarrow \infty} \eta=\beta$, and thus,

$$
\lim _{\gamma \rightarrow \infty} e^{\gamma} x^{*}(\gamma)=q^{*}
$$

As we have observed above, in addition to the symmetric one there are two asymmetric equilibria in the classical model. Hence, it is expected that the quantum model also possesses asymmetric equilibria at least as far as the entanglement is not too large. In fact, we can see that this conjecture is valid for the case of $a=3$ as follows. By substituting $x_{j}$ with $q_{j}$, equation (14) can be rewritten as

$$
B R_{j}: \quad\left(a+q_{j}-q_{i}-\left(q_{j}-a\right)^{3}\right) \cosh \gamma-e^{\gamma} q_{j}=0 .
$$

$B R_{j}$ is a locus of quantities, which is determined by firm $j$ 's best response strategy $x_{j}$ to the opponent's strategy $x_{i}$. Each intersection of $B R_{1}$ and $B R_{2}$ represents quantities produced in some equilibrium. Figure 1 depicts $B R_{1}$ and $B R_{2}$ for $\gamma=0,0.285$ and 0.6 , respectively. Figure 1 displays that the number of equilibria varies as $\gamma$ changes. Figure 1(i) corresponds to the classical model, where there are three equilibria. Figure 1(ii), in which five equilibria exist, shows the possibility that the number of equilibria increase by the existence of the entanglement. In figure 1(iii), asymmetric equilibria disappear and the only one symmetric equilibrium remains. Evidently from these figures, there is the possibility of multiple equilibria even if the entanglement exists. However, we can prove that asymmetric equilibria vanish when $\gamma$ goes large. 


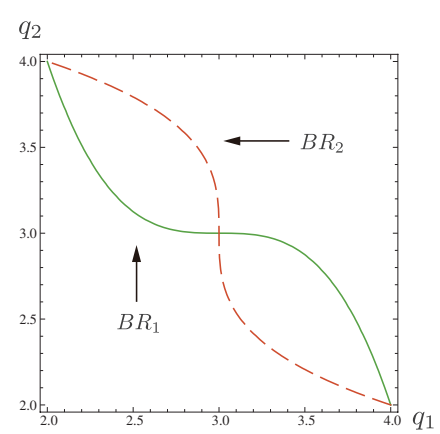

(i) $\gamma=0$

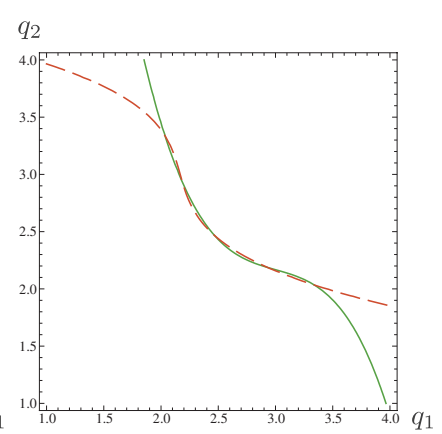

(ii) $\gamma=0.285$

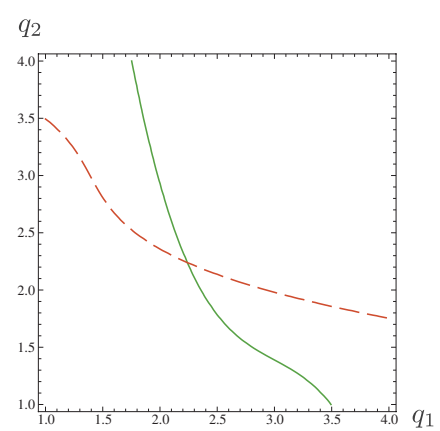

(iii) $\gamma=0.6$

Figure 1. $B R_{1}$ and $B R_{2}$ for $a=3$.

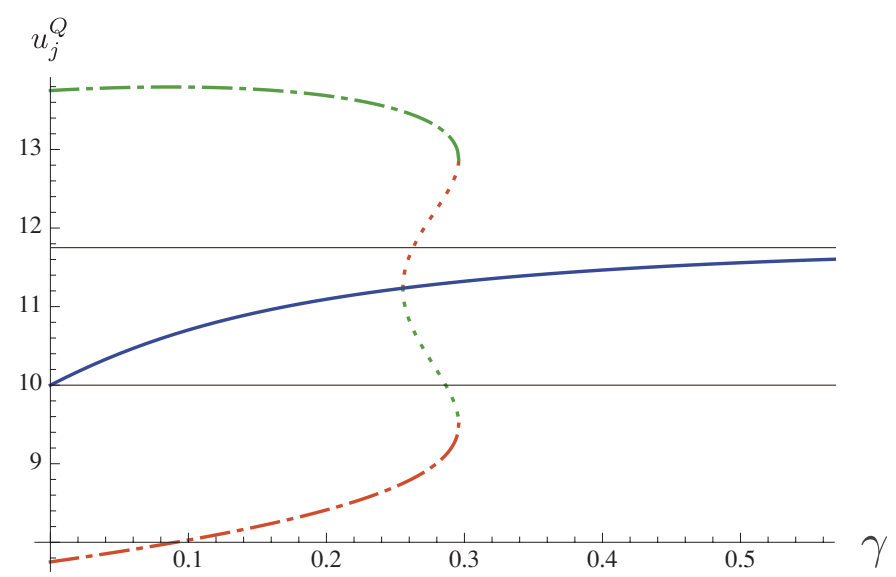

Figure 2. The profits at quantum Nash equilibria as a function of the entanglement parameter $\gamma$, where $a=3$ and $d=10$. The dash-dotted and dotted lines, colored green or red, represent the profits at the asymmetric equilibria. Each pair of lines, dash-dotted or dotted, corresponds to the profits gained at one of the asymmetric equilibria. For instance, when firm $j$ earns the profit indicated by the red dash-dotted (resp. dotted) line, the opponent earns the profit the green dash-dotted (resp. dotted) line points to, and vice versa. The blue line, depicted at the center, represents the profit at the unique symmetric equilibrium.

Proposition. For a sufficiently large $\gamma,\left(x^{*}(\gamma), x^{*}(\gamma)\right)$ is the unique equilibrium.

It is worth pointing out that the quantifier 'sufficiently large' in the proposition is not so restrictive by the following reason. To obtain the proposition, we use the fact that $\operatorname{sech} \gamma / e^{\gamma} \rightarrow 0$ as $\gamma \rightarrow \infty$. Since $\operatorname{sech} \gamma / e^{\gamma}$ converges very quickly, the lower bound for $\gamma$, which guarantees the uniqueness of equilibria, is not so large. For instance, any asymmetric equilibrium cannot exist for $\gamma>\gamma_{2} \simeq 0.296$ when $a=3$ (as we will see in figure 2).

Finally, we consider the transition of equilibria of the game from purely classical to fully quantum, as $\gamma$ increases from zero to infinity. Figure 2 depicts the transition process for the case of $a=3$ and $d=10$ : the number of equilibria changes, as $\gamma$ grows large, from 3 to 5, from 5 to 3 and from 3 to 1 at last. More precisely, there are two thresholds, namely $\gamma_{1} \simeq 0.255$ 
and $\gamma_{2} \simeq 0.296$ : for $0 \leqslant \gamma<\gamma_{1}$, there are three equilibria; for $\gamma_{1} \leqslant \gamma<\gamma_{2}$, there are five; for $\gamma=\gamma_{2}$, there are three; and for $\gamma_{2}<\gamma$, the symmetric (and unique) one remains. The horizontal line at $11.75(=7 / 4+d)$ represents the half of the maximum joint profit, and the horizontal line at 10 represents the profit at the symmetric Nash equilibrium of the classical Cournot game. As easily seen from the figure, asymmetric equilibria vanish and the unique symmetric equilibrium monotonically approaches to the optimal one as $\gamma$ goes large.

It is worth mentioning that in quantum prisoners' dilemma the thresholds in the entanglement parameter, which give rise to different equilibria, can depend on the strategy space available to the players and on the quantization method, as shown in [15]. Similar to [15], the thresholds in our model are sensitive to the choice of strategy space or the quantization method. For instance, one can easily check how the thresholds changes in a model where outputs are determined by $q_{i}=x_{i} \cosh \gamma+2 x_{j} \sinh \gamma$. However, we conjecture that our asymptotic result stated in the proposition is robust to those changes provided that changes are continuous in some sense. In particular, consider another quantized model such that outputs are determined by functions which are homotopic to equations (9) and (10). Then, there exists the unique symmetric equilibrium for sufficiently large entanglement parameters.

\section{Acknowledgments}

We would like to thank the anonymous referees for helpful comments and suggestions. All remaining errors are our own. We all acknowledge financial support from Grant-in-Aid for Creative Scientific Research no 19GS0101 of the Japan Society for the Promotion of Science. TS was also supported by Grant-in-Aid for Young Scientists (Start-up) no 20830095 of the Japan Society for the Promotion of Science.

\section{Appendix. Proof of the proposition}

Given a sufficiently large $\gamma$, let $\left(q_{1}, q_{2}\right)$ be equilibrium outputs. Suppose in contrast $q_{2}-q_{1}=\delta \neq 0$. Then, equation (17) implies

$$
\begin{aligned}
& \left(a-\delta-A^{3}\right) \cosh \gamma-e^{\gamma} q_{1}=0, \\
& \left(a+\delta-(A+\delta)^{3}\right) \cosh \gamma-e^{\gamma}\left(q_{1}+\delta\right)=0,
\end{aligned}
$$

where $A \equiv q_{1}-a$. Subtracting equation (A.1) from equation (A.2), we have

$$
(2 \delta-B) \cosh \gamma-\delta e^{\gamma}=0,
$$

where $B \equiv 3 A^{2} \delta+3 A \delta^{2}+\delta^{3}$. It implies

$$
B \cosh \gamma=\delta\left(2 \cosh \gamma-e^{\gamma}\right)=\delta e^{-\gamma} .
$$

Since we assume that $\delta \neq 0$, we have $B / \delta=e^{-\gamma} / \cosh \gamma$, that is,

$$
\delta^{2}+3 A \delta+3 A^{2}-\frac{\operatorname{sech} \gamma}{e^{\gamma}}=0
$$

It is necessary for equation (A.5) having a real solution that

$$
\frac{4}{3} \frac{\operatorname{sech} \gamma}{e^{\gamma}} \geqslant A^{2}
$$

which implies that $A$ must be sufficiently close to zero since sech $\gamma / e^{\gamma} \rightarrow 0$ as $\gamma \rightarrow \infty$. The solution of equation (A.5) is given by

$$
\delta=\frac{-3 A \pm \sqrt{-3 A^{2}+4 \frac{\operatorname{sech} \gamma}{e^{\gamma}}}}{2} \simeq 0 .
$$


On the other hand, equation (A.1) implies that

$$
\delta=a-A^{3}-q_{1} e^{\gamma} \operatorname{sech} \gamma=a-A^{3}-(A+a) e^{\gamma} \operatorname{sech} \gamma .
$$

Since $e^{\gamma} \operatorname{sech} \gamma \rightarrow 2$ and $A \rightarrow 0$ as $\gamma \rightarrow \infty$, we obtain

$$
\delta \simeq-a,
$$

which implies that $\delta$ is bounded away from zero, a contradiction. Thus, for a sufficiently large $\gamma$, any equilibrium must be symmetric. However, $\left(x^{*}(\gamma), x^{*}(\gamma)\right)$ is the unique symmetric solution of equation (14).

\section{References}

[1] Gibbons R 1992 Game Theory for Applied Economists (Princeton, NJ: Princeton University Press)

[2] Axelrod R 1984 The Evolution of Cooperation (New York: Basic Books)

[3] Maynard-Smith J 1982 Evolution and the Theory of Games (Cambridge: Cambridge University Press)

[4] Meyer D A 1999 Phys. Rev. Lett. 821052

[5] Eisert J, Wilkens M and Lewenstein M 1999 Phys. Rev. Lett. 833077

[6] Benjamin S C and Hayden P M 2001 Phys. Rev. A 64030301

[7] Arfi B 2005 Theory and Decision 59127

[8] Benjamin S C 2000 Phys. Lett. A 277180

[9] Du J, Li H, Xu X, Shi M, Zhou X and Han R 2001 Working paper arXiv:quant-ph/0103004v1

[10] Du J, Xu X, Li H, Zhou X and Han R 2001 Working paper arXiv:quant-ph/0010050v3

[11] Marinatto L and Weber T 2000 Phys. Lett. A 272291

[12] Marinatto L and Weber T 2000 Phys. Lett. A 277183

[13] Nawaz A and Toor A H 2004 J. Phys. A: Math. Gen. 374437

[14] Eisert J and Wilkens M 2000 J. Modern Optics 472543

[15] Flitney A P and Hollenberg L C L 2007 Phys. Lett. A 363381

[16] Ichikawa T and Tsutsui I 2007 Ann. Phys. 322531

[17] Ichikawa T, Tsutsui I and Cheon T 2008 J. Phys. A: Math. Theor. 41135303

[18] Toyota N 2003 Working paper arXiv:quant-ph/0307029v1

[19] Du J, Xu X, Li H, Zhou X and Han R 2001 Phys. Lett. A 2899

[20] Du J, Li H, Xu X, Zhou X and Han R 2003 J. Phys. A: Math. Gen. 366551

[21] Tirole J 1988 The Theory of Industrial Organization (Cambridge: MIT Press)

[22] Li H, Du J and Massar S 2002 Phys. Lett. A 30673

[23] Cournot A 1897 Researches into the Mathematical Principles of the Theory of Wealth (New York: Macmillan)

[24] Du J, Li H and Ju C 2003 Phys. Rev. E 68016124

[25] Du J, Ju C and Li H 2005 J. Phys. A: Math. Gen. 381559

[26] Lo C F and Kiang D 2004 Phys. Lett. A 32194

[27] Qin G, Chen X, Sun M and Du J 2005 J. Phys. A: Math. Gen. 384247

[28] Lo C F and Kiang D 2003 Phys. Lett. A 318333

[29] Lo C F and Kiang D 2005 Phys. Lett. A 34665

[30] Lo C F and Kiang D 2003 Europhys. Lett. 64592 